\title{
The First Boundary Value and Eigenvalue Problems for Degenerate Elliptic Equations, I
}

\author{
By \\ Kazumasa SuzUKI*
}

\section{Introduction}

The preceding paper [3] referred to the elliptic equation

$$
\sum_{i, j=1}^{m} \frac{\partial}{\partial x_{j}}\left(p_{i j} \frac{\partial u}{\partial x_{i}}\right)-q u=f
$$

which is degenerate on the boundary. In this paper we discuss the first boundary value and eigenvalue problems for the elliptic equations of the same form which may degenerate in the interior of the domain. The equations treated in this paper include as their special type uniformly elliptic equations.

We treat only weak solutions. However, we weaken the restriction on the coefficients. Our method to solve the problems owes to Sobolev [2], in which we find the boundary value and eigenvalue problems for the Laplace equation.

In $\S 1$ we arrange some inequalities to be used in the succeeding sections. Section 2 is devoted to some basic lemmas applied to a variational method. We solve, by a variational method, the first boundary value and eigenvalue problems in $\S 3$ and $\S 4$, respectively.

The author wishes to express his sincere thanks to Prof. M. Hukuhara for his helpful suggestions and constant encouragement.

\section{$\S 1$. Inequalities}

Let $\Omega$ be a bounded domain with definite measure in the $m$ -

Received November 21, 1967.

Communicated by M. Hukuhara.

* Gakushûin High School. 
dimensional Euclidean space $E$. We denote the space of measurable functions $u$ defined in $\Omega$ with

$$
\|u\|_{p} \equiv\left(\int_{\Omega}|u|^{p} d V\right)^{1 / p}<\infty
$$

by $L_{p}$ and the space of measurable functions $u$ defined in $\Omega$ with

$$
\|u\|_{\infty} \equiv \operatorname{vrai}_{\Omega} \max |u(x)|<\infty
$$

by $L_{\infty}$.

By $\dot{C}_{\infty}$ we denote the set of infinitely differentiable functions defined in $E$ with supports strictly contained in $\Omega$.

When we have

$$
\int_{\Omega} u \frac{\partial \zeta}{\partial x_{i}} d V=-\int_{\Omega} w_{i} \zeta d V
$$

for some function $u$ defined in $\Omega$ and for every function $\zeta \in \dot{C}_{\infty}$ we call $w_{i}$ the generalized derivative of $u$ with respect to $x_{i}$ and denote it by $\frac{\partial u}{\partial x_{i}}$.

By $W_{p}^{(1)}$ we denote the space of functions $u$ measurable in $\Omega$ and having the first order generalized derivatives also measurable in $\Omega$, and for which

$$
\|u\|_{W_{(p)}^{(1)}} \equiv\left[\int_{\Omega}\left\{\sum_{i=1}^{m}\left|\frac{\partial u}{\partial x_{i}}\right|^{p}+|u|^{p}\right\} d V\right]^{1 / p}<\infty .
$$

As is well known $W_{p}^{(1)}$ is complete.

We denote the closure of $\dot{C}_{\infty}$ in $W_{p}^{(1)}$ by $\dot{W}_{p}^{(1)}$. For $\varphi \in W_{p}^{(1)}$ we shall denote by $W_{p}^{(1)}$ the subset of $W_{p}^{(1)}$ defined by

$$
\stackrel{\varphi}{W}_{p}^{(1)} \equiv\left\{u \mid u-\varphi \in \dot{W}_{p}^{(1)}\right\}
$$

First we arrange some lemmas which give some inequalities in $W_{2}^{(1)}$.

Lemma 1. 1. When $m \geqq 2$, for any $u \in W_{2}^{(1)}$ we have

$$
\|u\|_{2 m /\{m(1+r)-2\}} \leqq B_{m r}\|\nabla u\|_{2 /(1+r)},
$$

where $0<r<1$ and $B_{m r}$ is some constant not depending on $u$. 
Proof. Take $\left\{u_{k} \in \dot{C}_{\infty}\right\}$ such that

$$
\left\|u-u_{k}\right\|_{W_{2}^{(1)} \rightarrow 0}(k \rightarrow \infty) .
$$

Obviously we have

$$
\begin{aligned}
& u_{k}(x)=\int_{-\infty}^{x_{i}} \frac{\partial u_{k}}{\partial x_{i}} d x_{i}=-\int_{x_{i}}^{\infty} \frac{\partial u_{k}}{\partial x_{i}} d x_{i}, \\
& \left|u_{k}(x)\right| \leqq \frac{1}{2} \int_{-\infty}^{\infty}\left|\frac{\partial u_{k}}{\partial x_{i}}\right| d x_{i} \leqq \frac{1}{2} \int_{-\infty}^{\infty}\left|\nabla u_{k}\right| d x_{i}
\end{aligned}
$$

and, therefore, we have

$$
\left|u_{k}(x)\right|^{m /(m-1)} \leqq\left(\frac{1}{2}\right)^{m /(m-1)} \prod_{i=1}^{m}\left(\int_{-\infty}^{\infty}\left|\nabla u_{k}\right| d x_{i}\right)^{1 /(m-1)} .
$$

From Hölder's inequality it follows that

$$
\begin{aligned}
& \int_{-\infty}^{\infty}\left|u_{k}\right|^{m /(m-1)} d x_{j} \leqq\left(\frac{1}{2}\right)^{m /(m-1)}\left(\int_{-\infty}^{\infty}\left|\nabla u_{k}\right| d x_{j}\right)^{1 /(m-1)} \\
& \quad \times \int_{-\infty}^{\infty}\left(\prod_{\substack{i=1 \\
i \neq j}}^{m} \int_{-\infty}^{\infty}\left|\nabla u_{k}\right| d x_{i}\right)^{1 /(m-1)} d x_{j} \\
& \leqq\left(\frac{1}{2}\right)^{m /(m-1)}\left(\int_{-\infty}^{\infty}\left|\nabla u_{k}\right| d x_{j}\right)^{1 /(m-1)} \\
& \quad \times\left(\prod_{\substack{i=1 \\
i \neq j}}^{m} \int_{-\infty}^{\infty} d x_{j} \int_{-\infty}^{\infty}\left|\nabla u_{k}\right| d x_{i}\right)^{1 /(m-1)} .
\end{aligned}
$$

In a similar way we have

$$
\begin{aligned}
& \int_{-\infty}^{\infty} d x_{1} \int_{-\infty}^{\infty} d x_{2} \cdots \int_{-\infty}^{\infty}\left|u_{k}\right|^{m /(m-1)} d x_{m} \\
& \leqq\left(\frac{1}{2}\right)^{m /(m-1)}\left(\int_{-\infty}^{\infty} d x_{1} \int_{-\infty}^{\infty} d x_{2} \cdots \int_{-\infty}^{\infty}\left|\nabla u_{k}\right| d x_{m}\right)^{m /(m-1)} .
\end{aligned}
$$

Thus we have

$$
\left\|u_{k}\right\|_{m /(m-1)} \leqq \frac{1}{2} i \nabla u_{k} \|_{1}
$$

Putting

$$
u_{k}=u_{k}^{(2 m-1) /\{m(1+r)-2\}}
$$

we get

$$
\left\|u_{k}\right\|_{2 m /\{m(1+r)-2\}} \leqq \frac{m}{m(1+r)-2} i \nabla u_{k} \|_{2 /(1+r)}
$$


Since we also get

$$
\left\|u_{k}-u_{k^{\prime}}\right\|_{2 m /(m(1-r)-2\}} \leqq \frac{m}{m(1+r)-2}\left\|\nabla\left(u_{k}-u_{k^{\prime}}\right)\right\| \nabla\left(u_{k}-u_{k^{\prime}}\right) \|_{2 /(1+r)}
$$

because of

$$
\begin{aligned}
& \left\|\nabla\left(u_{k}-u_{k^{\prime}}\right)\right\|_{2 /(1+r)} \leqq(\operatorname{mes} \Omega)^{r / 2} \mid\left\|\nabla\left(u_{k}-u_{k^{\prime}}\right)\right\|_{2} \\
& \leqq(\operatorname{mes} \Omega)^{r / 2}|| u_{k}-u_{k^{\prime}} \|_{W_{2}^{(1)} \rightarrow 0 \quad\left(k, k^{\prime} \rightarrow \infty\right),}
\end{aligned}
$$

we have

$$
\|u\|_{2 m /\{m(1+r)-2\}} \leqq \frac{m}{m(1+r)-2} \|\left.\nabla u\right|_{2 /(1+r)} .
$$

Obviously from (1) and (2) follow the following corollaries.

Corollary 1. When $m=1$, for any $u \in W_{2}^{(1)}$ we have

$$
\|u\|_{\infty} \leqq \frac{1}{2}\|\nabla u\|_{1} \text {. }
$$

Corollary 2. When $m \geqq 2$ for any $u \in W_{2}^{(1)}$ we have

$$
\|u\|_{m /(m-1)} \leqq \frac{1}{2}\|\nabla u\|_{1} .
$$

Lemma 1.2. For any $u \in W_{2}^{(1)}$ we have

$$
\|u\|_{2 /(1+r)} \leqq B_{m r}{ }^{\prime} \|\left.\nabla u\right|_{2 /(1+r)},
$$

where $0 \leqq r<1$ and $B_{m r}{ }^{\prime}$ is some constant not depending on $u$.

Proof. In the case $m \geqq 3$ or $m=2, r \neq 0$, by Hölder's inequality we have

$$
\|u\|_{2 /(1+r)} \leqq\left.(\operatorname{mes} \Omega)^{1 / m}|| u\right|_{2 m /(m(1+r)-2\}}
$$

and therefore we have

$$
\|\left. u\right|_{2 /(1+r)} \leqq\left. B_{m r}|| \nabla u_{i}\right|_{2(1+r)} \quad \text { (cf. Lemma 1.1). }
$$

In the case $m=2, r=0$, from Corollary 2 to Lemma 1.1 follows

$$
\|u\|_{2 /(1+r)} \leqq \frac{1}{2}(\operatorname{mes} \Omega)^{1 / 2}|| \nabla u \|_{2 /(1+r)} .
$$

In the case $m=1$, by Corollary 1 to Lemma 1.1 we have 


$$
\|u\|_{2 /(1+r)} \leqq \frac{1}{2} \operatorname{mes} \Omega\|\nabla u\|_{2 /(1+r)}
$$

Lemma 1. 3. For any $u \in W_{2}^{(1)}$ we have

$$
\|u\|_{2} \leqq B_{m r}{ }^{\prime \prime}|| u\left\|_{2 /(1+r)}^{1-p_{m r}}\right\| \nabla u \|_{2 /(1+r)}^{p_{m r}},
$$

where $0 \leqq r<\min \left\{\frac{2}{m}, 1\right\}$ and $p_{m r}\left(0<p_{m r}<1\right), B_{m r}$ " are some constants not depending on $u$.

Proof. In the case $m \geqq 3$ or $m=2, r \neq 0$, from Hölder's inequality follows

$$
\begin{aligned}
& \int_{\Omega} u^{2} d V=\int_{\Omega} u^{\alpha} u^{2-\infty} d V \\
& \leqq\left(\int_{\Omega} u^{2 /(1+r)} d V\right)^{\omega_{(1+r}(1+2)}\left(\int_{\Omega} u^{(2-\infty)} \frac{2}{2-\alpha(1+r)} d V\right)^{\left\{2-\alpha_{(1 \mid r)\} / 2}\right.}
\end{aligned}
$$

for every $\alpha$ such that $0<\alpha<\frac{2}{1+r}$. Putting $\alpha=2-m r$, we have

$$
\|u\|_{2}^{2} \leqq i \mid u\left\|_{2 /(1+r)}^{2-m r}\right\| u \|_{2 m /\{m(1+r)-2\}}^{m r}
$$

and therefore we have

$$
\|u\|_{2}^{2} \leqq B_{m r}{ }^{m r}|| u\left\|_{2 /(1+r)}^{2-m r}\right\| \nabla u \|_{2 /(1+r)}^{m r}
$$

(cf. Lemma 1.1).

In the case $m=2, r=0$, by Corollary 2 to Lemma 1.1 we have

$$
\begin{aligned}
\|u\|_{2}^{2} & \leqq \frac{1}{2}\|u\|_{2}\|\nabla u\|_{1} \\
& \leqq \frac{1}{2}(\operatorname{mes} \Omega)^{1 / 2}\|u\|_{2}\|\nabla u\|_{2} \\
& =\frac{1}{2}(\operatorname{mes} \Omega)^{1 / 2}\|u\|_{2 /(1+r)} i \mid \nabla u \|_{2 /(1+r)} .
\end{aligned}
$$

In the case $m=1$, by Corollary 1 to Lemma 1.1 we have

$$
\begin{aligned}
& \int_{\Omega} u^{2} d V=\int_{\Omega} u^{2 /(1+r)} u^{2\{1-1 /(1+r)\}} d V \\
& \leqq \int_{\Omega} u^{2 /(1+r)} d V\left\{\frac{1}{2}(\operatorname{mes} \Omega)^{(1-r) / 2}\|\nabla u\|_{2 /(1+r)}\right\}^{2 r /(1+r)} \\
& \leqq\left\{\frac{1}{2}(\operatorname{mes} \Omega)^{(1-r) / 2}\right\}^{2 r /(1+r)}\|u\|_{2 /(1+r)}^{2 /(1+r)}\|\nabla u\|_{2 /(1+r)}^{2 r /(1+r)}
\end{aligned}
$$


Lemma 1. 3'. When $m \geqq 2$ for any $u \in \stackrel{\circ}{W}_{2}^{(1)}$ we have

$$
\|u\|_{m(2-r) /(m-1)} \leqq\left. B_{m r}{ }^{\prime \prime \prime}|| u_{\|_{2}}^{\prime \prime}\right|_{m r} ^{1-p_{m}}\|\nabla u\|_{2 /(1+r)}^{p_{m r^{\prime}}},
$$

where $0 \leqq r<1$ and $p_{m r}{ }^{\prime}\left(0<p_{m r}{ }^{\prime}<1\right), B_{m r}{ }^{\prime \prime}$ are some constants not depending on $u$.

Proof. Putting $u=u^{\infty}$ in the inequality in Corollary 2 to Lemma 1. 1, we have

$$
\begin{aligned}
& \left\|u^{\alpha}\right\|_{m /(m-1)} \leqq \frac{\alpha}{2}\left\|u^{\alpha-1} \nabla u\right\|_{1} \\
& \leqq \frac{\alpha}{2}\|\nabla u\|_{2 /(1+r)}\|u\|_{2(\alpha-1) /(1-r)}^{\alpha-1}
\end{aligned}
$$

for every $\alpha$ such that $\alpha>1$. If we put $\alpha=2-r$, then we get

$$
\|u\|_{m(2-r) /(m-1)}^{2-r} \leqq \frac{2-r}{2}\|\nabla u\|_{2 /(1+r)}\|u\|_{2}^{1-r}
$$

Lemma 1. 4. If a subset $\mathfrak{A}$ in $\stackrel{\circ}{2 /(1+r)}_{(1)}$ is bounded in $W_{2 /(1+r)}^{(1)}$, then $\mathfrak{A}$ is relatively compact in $L_{2}$, where $0 \leqq r<\min \left\{\frac{2}{m}, 1\right\}$.

Proof. As is well known, $\mathfrak{A}$ is relatively compact in $L_{2 /(1+r)}$. Taking a Cauchy sequence $\left\{u_{k} \in \mathfrak{A}\right\}$ such that

$$
\left\|u_{k}-u_{k^{\prime}}\right\|_{2 /(1+r)} \rightarrow 0\left(k, k^{\prime} \rightarrow \infty\right),
$$

from Lemma 1.3 follows

$$
\begin{aligned}
& \left\|u_{k}-u_{k^{\prime}}\right\|_{2} \leqq B_{m r}{ }^{\prime \prime}\left\|u_{k}-u_{k^{\prime}}\right\|_{2 /(1+r)}^{1-p_{m r}} \mid i \nabla\left(u_{k}-u_{k^{\prime}}\right) \|_{2 /(1+r)}^{p_{m r}} \\
\rightarrow & 0\left(k, k^{\prime} \rightarrow \infty\right) .
\end{aligned}
$$

Therefore $\mathfrak{A}$ is relatively compact in $L_{2}$. Q.E.D.

Next we define some functionals on $W_{2}^{(1)}$ as follows :

$$
\begin{aligned}
& D[u, v] \equiv \int_{\Omega} \sum_{i, j=1}^{m} p_{i j} \frac{\partial u}{\partial x_{i}} \frac{\partial v}{\partial x_{j}} d V, \\
& H_{\rho}[u, v] \equiv \int_{\Omega} \rho u v d V, \\
& D[u] \equiv D[u, u], \quad H_{\rho}[u] \equiv H_{\rho}[u, u],
\end{aligned}
$$

where $u, v \in W_{2}^{(1)}$.

We shall give some inequalities with respect to these functionals. 
Let $\left(p_{i j}\right)$ satisfy

$$
0 \leqq p_{0}(x) \sum_{i=1}^{m} \xi_{i}{ }^{2} \leqq \sum_{i}^{m} p_{j=1}^{m} p_{i j} \xi_{i} \xi_{j} \leqq p_{1}(x) \sum_{i=1}^{m} \xi_{i}{ }^{2} \quad \text { in } \Omega,
$$

where $\left(\xi_{1}, \cdots, \xi_{m}\right)$ is any real vector, $p_{0}{ }^{-1} \in L_{1 / r}(r \geqq 0)$ and $p_{1} \in L_{\infty}$. Hereafter, when this condition is satisfied we say that $\left(p_{i j}\right)$ has the degeneracy of the $r$-th degree.

Lemma 1.5. Assume that $\left(p_{i j}\right)$ has the degeneracy of the $r$-th degree.

Then for any $u \in W_{2}^{(1)}$ we have

$$
\left\|p_{0}^{-1}\right\|_{1 / r}^{-1}\|\nabla u\|_{2 /(1+r)}^{2} \leqq D[u]<\infty,
$$

where $0 \leqq r<1$ (when $r=0$, we read $1 / r=\infty$ ).

Proof. From Hölder's inequality it follows that

$$
\begin{aligned}
& \|\nabla u\|_{2 /(1+r)}^{2}=\left[\int_{\Omega}\left(\frac{1}{p_{0}}\right)^{1 /(1+r)}\left\{\sum_{i=1}^{m} p_{0}\left(\frac{\partial u}{\partial x_{i}}\right)^{2}\right\}^{1 /(1+r)} d V\right]^{1+r} \\
& \leqq\left\|p_{0}^{-1}\right\|_{1 / r} \int_{\Omega} \sum_{i, j=1}^{m} p_{i j}\left(\frac{\partial u}{\partial x_{i}}\right)\left(\frac{\partial u}{\partial x_{j}}\right) d V .
\end{aligned}
$$

The finiteness of $D[u]$ is evident.

Lemma 1. 6. Assume that

$$
\rho \in L_{r_{1}} \quad\left(r_{1}=\left\{\begin{array}{cl}
\frac{m}{2-m r} & (m \geqq 3 \text { or } m=2, r \neq 0) \\
\infty & (m=2, r=0) \\
1 & (m=1)
\end{array}\right)\right.
$$

and

$$
\phi \in W_{2}^{(1)} \cap L_{r_{2}} \quad\left(r_{2}=\left\{\begin{array}{cl}
\frac{2 m}{m(1+r)-2} & (m \geqq 3 \text { or } m=2, r \neq 0) \\
2 & (m=2, r=0) \\
\infty & (m=1)
\end{array}\right)\right.
$$

where $0 \leqq r<\min \left\{\frac{2}{m}, 1\right\}$.

Then for any $u \in \stackrel{\varphi}{W}_{2}^{(1)}$ we have

$$
\left|H_{\rho}[u]\right| \leqq\|\rho\|_{r_{1}}\left(C_{m r} \mid\|\nabla(u-\phi)\|_{2 /(1 \mid r)}+\|\rho\|_{r_{2}}\right)^{2},
$$

where $C_{m r}$ is some constant not depending on $u$. 
Proof. When $m \geqq 3$ or $m=2, r \neq 0$, by Hölder's inequality we have

$$
\begin{aligned}
& \left|H_{\rho}[u]\right| \leqq \int_{\Omega}|\rho| u^{2} d V \\
& \leqq\|\rho\|_{m /(2-m r)}\|u\|_{2 m /\{m(1+r)-2\}}^{2} \\
& \leqq\|\rho\|_{m /(2-m r)}\left(\|u-\varphi\|_{2 m /\{m(1+r)-2\}}+\|\varphi\|_{2 m /(m(1+r)-2\}}\right)^{2} \\
& \leqq\|\rho\|_{r_{1}}\left(B_{m r}\|\nabla(u-\varphi)\|_{2 /(1+r)}+\|\rho\|_{2 m /\{m(1+r)-2\}}\right)^{2}
\end{aligned}
$$

(cf. Lemma 1. 1).

When $m=1, r=0$, we have

$$
\begin{aligned}
& \left|H_{\rho}[u]\right| \leqq \int_{\Omega}|\rho| u^{2} d V \leqq\|\rho\|_{\infty}\|u\|_{2}^{2} \\
& \leqq\|\rho\|_{r_{1}}\left(\|u-\varphi\|_{2 /(1+r)}+\|\left.\varphi\right|_{i_{2}}\right)^{2} \\
& \leqq\|\rho\|_{r_{1}}\left(B_{m r}{ }^{\prime}\|\nabla(u-\varphi)\|_{2 /(1+r)}+\|\varphi\|_{2}\right)^{2}
\end{aligned}
$$

(cf. Lemma 1.2).

When $m=1$, we have

$$
\begin{aligned}
& \left|H_{\rho}[u]\right| \leqq \int_{\Omega}|\rho| u^{2} d V \\
& \leqq\|\rho\|_{1}\left\|u^{2}\right\|_{\infty} \leqq\|\rho\|_{1}\left(\|u-\varphi\|_{\infty}+\|\varphi\|_{\infty}\right)^{2} \\
& \leqq\|\rho\|_{1}\left(\frac{1}{2}\|\nabla(u-\varphi)\|_{1}+\|\varphi\|_{\infty}\right)^{2}
\end{aligned}
$$

(cf. Corollary 1 to Lemma 1.1)

$$
\leqq\|\rho\|_{r_{1}}\left(\frac{1}{2}(\operatorname{mes} \Omega)^{(1-r) / 2}\|\nabla(u-\varphi)\|_{2 /(1+r)}+\|\varphi\|_{\infty}\right)^{2} .
$$

Lemma 1.6'. Assume that

$$
\rho \in L_{r_{3}} \quad\left(r_{3}=\left\{\begin{array}{l}
\frac{m(2-r)}{2-m r}(m \geqq 2) \\
1+\varepsilon(\varepsilon \text { is some positive constant })(m=1)
\end{array}\right),\right.
$$

where $0 \leqq r<\min \left\{\frac{2}{m}, 1\right\}$.

Then for any $u \in W_{2}^{(1)}$ we have

$$
\left|H_{\rho}[u]\right| \leqq C_{m r}{ }^{\prime}\|\rho\|_{r_{3}}\|u\|_{2}^{2\left(1-p_{m r}{ }^{\prime \prime)}\right.}\|\nabla u\|_{2 /(1+r)}^{2 p_{m}{ }^{\prime \prime}},
$$

where $p_{m r}{ }^{\prime \prime}\left(0<p_{m r}{ }^{\prime \prime}<1\right)$ and $C_{m r}^{\prime}$ are some constants not depending on $u$. 
Proof. When $m \geqq 2$, by Hölders inequality we have

$$
\begin{aligned}
& \left|H_{\rho}[u]\right| \leqq \int_{\Omega}|\rho| u^{2} d V \\
& \leqq\left.\|\rho\|_{m(2-r) /(2-m r)}|| u\right|_{m(2-r) /(m-1)} ^{2} .
\end{aligned}
$$

From Lemma 1. $3^{\prime}$ follows

$$
\left|H_{\rho}[u]\right| \leqq B_{m r^{\prime \prime 2}}\|\rho\|_{r_{3}}\|u\|_{2}^{2\left(1-p_{m r^{\prime}}{ }^{\prime}\right.}|| \nabla u \|_{2 /(1+r)}^{2 p_{m r^{\prime}}} .
$$

When $m=1$, we have

$$
\begin{aligned}
& \left|H_{\rho}[u]\right| \leqq\left\|_{\rho} \rho\right\|_{r_{3}}\|u\|_{2 r_{3} /\left(r_{3}-1\right)}^{2} \\
& =\|\rho\|_{r_{3}}\|u\|_{2}^{2\left(r_{3}-1\right) / r_{3}}\|u\|_{\infty}^{2 / r_{3}} \\
& \leqq \mid i \rho\left\|_{r_{3}}\right\| u\left\|_{2}^{2\left(r_{3}-1\right) / r_{3}} \times \frac{1}{2}\right\| \nabla u \|_{1}^{2 / r_{3}}
\end{aligned}
$$

(cf. Corollary 1 to Lemma 1.1)

$$
\leqq\left\{\frac{1}{2}(\operatorname{mes} \Omega)^{(1-r) / 2}\right\}^{2 / r_{3}}\|\rho\|_{r_{3}}\|u\|_{2}^{2\left(r_{3}-1\right) / r_{3}}\|\nabla u\|_{2 /(1+r)}^{2 / r_{3}} .
$$

Lemma 1. 7. Assume that

$$
f \in L_{r_{4}} \quad\left(r_{4}=\left\{\begin{array}{cl}
\frac{2 m}{m(1-r)+2} & (m \geqq 3 \text { or } m=2, r \neq 0) \\
2 & (m=2, r=0) \\
1 & (m=1)
\end{array}\right),\right.
$$

and

$$
\varphi \in W_{2}^{(1)} \cap L_{r_{2}} \quad\left(r_{2}=\left\{\begin{array}{cl}
\frac{2 m}{m(1+r)-2} & (m \geqq 3 \text { or } m=2, r \neq 0) \\
2 & (m=2, r=0) \\
\infty & (m=1)
\end{array}\right),\right.
$$

where $0 \leqq r<1$.

Then for any $u \in \stackrel{\varphi}{W}_{2}^{(1)}$ we have

$$
|(f, u)| \leqq\|f\|_{r_{4}}\left(C_{m r}{ }^{\prime \prime !} \mid \nabla(u-\varphi)\left\|_{2 /(1+r)}+\right\| \varphi_{1} \|_{r_{2}}\right),
$$

where

$$
(f, u)=\int_{\Omega} f u d V .
$$

Proof. When $m \geqq 3$, or $m=2, r \neq 0$ from Hölder's inequality follows 


$$
\begin{aligned}
& |(f, u)| \leqq\|f\|_{2 m /\{m(1-r)+2\}} \mid\|u\|_{2 m /\{m(1+r)-2\}} \\
& \leqq\|f\|_{2 m /\{2-m(r-1)\}}\left(\|u-\varphi\|_{2 m /\{m(1+r)-2\}}+\|\varphi\|_{2 m /\{m(1+r)-2\}}\right) \\
& \leqq\|f\|_{2 m /\{2-m(r-1)\}}\left(B_{m r}\|\nabla(u-\varphi)\|_{2 /(1+r)}+\|\varphi\|_{2 m /\{m(1+r)-2\}}\right)
\end{aligned}
$$

(cf. Lemma 1.1).

When $m=2, r=0$ from Schwartz's inequality follows

$$
\begin{aligned}
& |(f, u)| \leqq\|f\|_{2}\|u\|_{2} \\
& \leqq\|f\|_{2}\left(\|u-\varphi\|_{2 /(1+r)}+\|\varphi\|_{2}\right) \\
& \leqq\|f\|_{r_{4}}\left(B_{m r}{ }^{\prime}\|\nabla(u-\varphi)\|_{2 /(1+r)}+\|\varphi\|_{2}\right) \quad \text { (cf. Lemma 1. 2). }
\end{aligned}
$$

When $m=1$, by Corollary 1 to Lemma 1.1 and Hölders inequality we have

$$
\begin{aligned}
& |(f, u)| \leqq\|f\|_{1}\|u\|_{\infty} \\
& \leqq\|f\|_{1}\left(\|u-\varphi\|_{\infty}+\|\varphi\|_{\infty}\right) \\
& \leqq\|f\|_{1}\left(\frac{1}{2}\|\nabla(u-\varphi)\|_{1}+\|\varphi\|_{\infty}\right) \\
& \leqq\|f\|_{1}\left(\frac{1}{2}(\operatorname{mes} \Omega)^{(1-r) / 2}\|\nabla(u-\varphi)\|_{2 /(1+r)}+\|\varphi\|_{\infty}\right) .
\end{aligned}
$$

\section{§. Completion of $W_{2}^{(1)}$}

If $\left(p_{i j}\right)=\left(p_{j i}\right)$ has the degeneracy of the $r$-th degree, the functional $\sqrt{D[\cdot]}$ plays the part of a norm on $W_{2}^{(1)}$. We denote by $D \dot{W}_{2}^{(1)}$ the completion of $\stackrel{\circ}{W}_{2}^{(1)}$ with respect to $\sqrt{D[\cdot]}$. For $\phi \in W_{2}^{(1)}$ we define

$$
D \stackrel{\varphi}{W}(1)=\left\{u \mid u-\varphi \in D \stackrel{\circ}{W}_{2}^{(1)}\right\} .
$$

When $u \in D \stackrel{\varphi}{W}_{2}^{(1)}\left(0 \leqq r<\min \left(\frac{2}{m}, 1\right)\right)$, take a corresponding Cauchy sequence $\left\{u_{k} \in \stackrel{\varphi}{W}_{2}^{(1)}\right\}$. Since $D\left[u_{k}-u_{k^{\prime}}\right] \rightarrow 0\left(k, k^{\prime} \rightarrow \infty\right)$, we have $\left\|u_{k}-u_{k^{\prime}}\right\|_{W_{2 /(1+r)}^{(1)}} \rightarrow 0\left(k, k^{\prime} \rightarrow \infty\right)$ (cf. Lemma 1.5 and Lemma 1.2) and therefore we have $\| u_{k}-\left.u_{k^{\prime}}\right|_{2} \rightarrow 0\left(k, k^{\prime} \rightarrow \infty\right)$ (cf. Lemma 1.3). Thus $u$ is identified with some function $u^{\prime}$ belonging to $W_{2 /(1+r)}^{(1)} \cap L_{2}$. The preceding lemmas keep valid for $D \stackrel{\varphi}{W}(1)$ (or $D \mathscr{W}_{2}^{(1)}$ ) in place of $\stackrel{\varphi}{W}_{2}^{(1)}$ (or $\stackrel{\circ}{W}_{2}^{(1)}$ ).

Lemma 2.1. Assume that $\left(p_{i j}\right)=\left(p_{j i}\right)$ has the degeneracy of the $r$-th degree and that 


$$
\begin{aligned}
& q \geqq 0, q \in u_{r_{1}} \quad\left(r_{1}=\left\{\begin{array}{cl}
\frac{m}{2-m r}(m \geqq 3 \text { or } m=2, r \neq 0) \\
\infty \quad(m=2, r=0) \\
1 \quad(m=1)
\end{array}\right)\right. \\
& f \in L_{r_{4}} \quad\left(r_{4}=\left\{\begin{array}{cl}
\frac{2 m}{m(1-r)+2} & (m \geqq 3 \text { or } m=2, r \neq 0) \\
2 & (m=2, r=0) \\
1 & (m=1)
\end{array}\right)\right. \\
& \varphi \in W_{2}^{(1)} \cap L_{r_{2}}\left(r_{2}=\left\{\begin{array}{cl}
\frac{2 m}{m(1+r)-2} & (m \geqq 3 \text { or } m=2,(r \neq 0) \\
2 & (m=2, r=0) \\
\infty & (m=1)
\end{array}\right)\right.
\end{aligned}
$$

where $r$ is some constant with $0 \leqq r<\min \left\{\frac{2}{m}, 1\right\}$.

Then we have

$$
-\infty<\inf _{\substack{\varphi \\ D W_{2}^{(1)}}}\left(D[u]+H_{q}[u]+2(f, u)\right)<\infty .
$$

Proof. From Lemmas 1.5, 1.6 and 1.7 follows

$$
\inf _{\substack{\varphi \\ D W_{2}^{(1)}}}\left(D[u]+H_{q}[u]+2(f, u)\right)<\infty .
$$

Again by these lemms we have

$$
\begin{aligned}
& D[u]+H_{q}[u]+2(f, u) \\
& \geqq\left\|p_{0}{ }^{-1}||_{1 / r}^{-1}|| \nabla u\right\|_{2 /(1+r)}^{2}-2\|f\|_{r_{4}}\left(C_{m r}{ }^{\prime \prime}\|\nabla(u-\varphi)\|_{2 /(1 \mid r)}+\|\varphi\|_{r_{2}}\right) \\
& \geqq\left\|p_{0}{ }^{-1}\right\|_{1 / r}^{-1}|| \nabla u\left\|_{2 /(1+r)}^{2}-2 C_{m r}{ }^{\prime \prime}\right\| f\left\|_{r_{4}}\right\| \nabla u \|_{2 /(1+r)} \\
& -2\|f\|_{r_{4}}\left(C_{m r}{ }^{\prime \prime}\|\nabla \varphi\|_{2 /(1+r)}+\|\varphi\|_{r_{2}}\right) \\
& \geqq\left.\left\|p_{0}{ }^{-1}\right\|\right|_{1 / r} ^{-1}|| \nabla u\left\|_{2 /(1+r)}^{2}-\varepsilon^{-1} C_{m r}{ }^{\prime \prime 2}|| f\right\|_{r_{4}}^{2}-\varepsilon \|\left.\nabla u\right|_{\left.\right|_{2} /(1+r)} ^{2} \\
& -2\|f\|_{r_{4}}\left(C_{m r}{ }^{\prime \prime}\|\nabla \varphi\|_{/ 2(1+r)}+\|\left.\varphi\right|_{i_{2}}\right) \\
& =\left(\left\|p_{0}^{-1}\right\|_{1 / r}^{-1}-\varepsilon\right)\|\nabla u\|_{2 /(1+r)}^{2}-\varepsilon^{-1} C_{m r}{ }^{\prime \prime}\|f\|_{r_{4}}^{2} \\
& -2\|f\|_{r_{4}}\left(C_{m r}{ }^{\prime \prime}\|\nabla \varphi\|_{2 /(1+r)}+\|\varphi\|_{r_{2}}\right) \\
& \geqq C,
\end{aligned}
$$

where $\varepsilon$ is some constant with $0<\varepsilon<\left\|p_{0}{ }^{-1}\right\|_{1 / r}^{-1}$ and $C$ is some constant not depending on $u$.

Lemma 2.2. Assume that $\left(p_{i j}\right)=\left(p_{j i}\right)$ has the degeneracy of the $r$-th degree and that 


$$
\begin{aligned}
& \rho \geqq \rho_{0}>0 \\
& \rho, q \in L_{r_{3}}\left(r_{3}=\left\{\begin{array}{l}
\frac{m(2-r)}{2-m r}(m \geqq 2) \\
1-\varepsilon(\varepsilon \text { is some positive constant })(m=1)
\end{array}\right),\right.
\end{aligned}
$$

where $r$ is some constant with $0 \leqq r<\min \left\{\frac{2}{m}, 1\right\}$.

Then we have

$$
-\infty<\inf _{\mathfrak{w}_{1}}\left(D[u]+H_{q}[u]\right)<\infty,
$$

where

$$
\mathfrak{W}_{1}=\left\{u \in D \dot{W}_{2}^{(1)} \mid H_{\rho}[u]=1\right\} \text {. }
$$

Proof. From Lemmas 1.5 and $1.6^{\prime}$ follows

$$
\inf _{\mathfrak{w}_{1}}\left(D[u]+H_{q}[u]\right)<\infty \text {. }
$$

Note that from Young's inequality follows

$$
\begin{aligned}
& \|u\|_{2}^{2\left(1-p_{m r}{ }^{\prime \prime}\right)}\|\nabla u\|_{2 /(1+r)}^{2 p_{m r}{ }^{\prime \prime}} \leqq\left(1-p_{m r}{ }^{\prime \prime}\right) \varepsilon^{-1 /\left(1-p_{m r}{ }^{\prime \prime}\right)} \| u_{{ }_{1}}^{\mid 2} \\
& +p_{m r}{ }^{\prime \prime} \varepsilon^{1 / p_{m r}{ }^{\prime \prime}}|| \nabla u \|_{2 /(1+r)}^{2},
\end{aligned}
$$

and that for $u \in \mathfrak{W}_{1}$ we have

$$
|i u|_{2}^{2} \leqq \rho_{0}{ }^{-1} H_{\rho}[u]=\rho_{0}^{-1} \text {. }
$$

By Lemmas 1.5 and $1.6^{\prime}$ we have

$$
\begin{aligned}
& D[u]+H_{q}[u] \geqq\left.\left\|\left.p_{0}^{-1}\right|_{1 / r} ^{-1}\right\| \nabla u\right|_{2 /(1+r)} ^{2} \\
& -C_{m r}{ }^{\prime}\|q\|_{r_{3}}\left\|\left.u\right|_{i_{2}} ^{2\left(1-p_{m r}{ }^{\prime \prime}\right.}\right\| \nabla u \|_{2 /(1+r)}^{2 p_{m r}{ }^{\prime \prime}} \\
& \geqq\left\|p_{0}{ }^{-1}\right\|_{1 / r}^{-1}\|\nabla u\|_{2(1 / r)}^{2}-C_{m r}{ }^{\prime}\|q\|_{r_{3}}\left(p_{m r}{ }^{\prime \prime} \varepsilon^{1 / p_{m r}}{ }^{\prime \prime}|| \nabla u \|_{{ }_{2} /(1+r)}^{2}\right. \\
& \left.+\left(1-p_{m r}{ }^{\prime \prime}\right) \varepsilon^{-1 /\left(1-p_{m r}{ }^{\prime \prime}\right)} \rho_{0}{ }^{-1}\right) \\
& =\left(\left\|p_{0}{ }^{-1}\right\|_{1 / r}^{-1}-\varepsilon^{1 / p_{m r}}{ }^{\prime \prime} C_{m r}{ }^{\prime} p_{m r}{ }^{\prime \prime}\|q\|_{r_{3}}\right)\|\nabla u\|_{2 /(1+r)}^{2} \\
& -\varepsilon^{-1 /\left(1-p_{m r}{ }^{\prime \prime}\right)} \rho_{0}{ }^{-1} C_{m r}\left(1-p_{m r}{ }^{\prime \prime}\right)\|q\|_{r_{4}} \\
& \geqq C^{\prime} \text {, }
\end{aligned}
$$

where $\varepsilon$ is some constant with $0<\varepsilon<\left(C_{m r}{ }^{\prime} p_{m r}{ }^{\prime \prime}\left\|p_{0}{ }^{-1}\right\|_{1 / r}\|q\|_{r_{3}}\right)^{-p_{m r}{ }^{\prime \prime}}$ and $C^{\prime}$ is some constant not depending on $u$.

Lemma 2. 3. Under the same assumptions as in Lemma 2.2, if we have

$$
D[u]+H_{q}[u] \leqq C
$$


for some $u \in \mathfrak{W}_{1}$, then we have

$$
D[u] \leqq C^{\prime},
$$

where $C^{\prime}$ is some constant not depending on $u$.

Proof. From the the condition of the lemma follows

$$
\begin{aligned}
& D[u] \leqq C+\left|H_{q}[u]\right| \\
& \leqq C+C_{m r}{ }^{\prime}\|q\|_{r_{3}}\|u\|_{2}^{2\left(1-p_{m r}{ }^{\prime \prime)}\right.}\|\nabla u\|_{2 /(1+r)}^{2 p_{m r}^{\prime \prime}} \quad \text { (cf. Lemma 1. } 6^{\prime} \text { ) } \\
& \leqq C+\left.C_{m r}{ }^{\prime}|| q\right|_{r_{3}} ^{i}\left(p_{m r}{ }^{\prime \prime} \varepsilon^{1 / p_{m r}}{ }^{\prime \prime}|| \nabla u \|_{2 /(1+r)}^{2}+\left(1-p_{m r}{ }^{\prime \prime}\right) \varepsilon^{-1 /\left(1-p_{m r}{ }^{\prime \prime} \rho_{0}{ }^{-1}\right)}\right. \\
& \leqq C+\varepsilon^{1 / p_{m r}}{ }^{\prime \prime} C_{m r}{ }^{\prime} p_{m r}{ }^{\prime \prime}\|q\|_{r_{3}}\left\|_{i} p_{0}^{-1}\right\|_{1 / r}^{-1} D[u] \\
& +\varepsilon^{-1 /\left(1-p_{m r}{ }^{\prime \prime}\right.}\left(1-p_{m r}{ }^{\prime \prime}\right) \rho_{0}{ }^{-1} C_{m r}{ }^{\prime}\|q\|_{r_{3}} \text {, }
\end{aligned}
$$

where $\varepsilon$ is any positive constant. Choosing $\varepsilon$ so small that

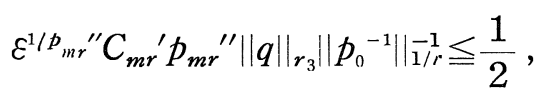

we have

$$
D[u] \leqq 2 C+2 \varepsilon^{-1\left(1-p_{m r}{ }^{\prime \prime}\right.}\left(1-p_{m r}{ }^{\prime \prime}\right) \rho_{0}{ }^{-1} C_{m r}{ }^{\prime}\|q\|_{r_{3}} .
$$

\section{§3. Boundary Value Problem}

Consider the boundary value problem for the degenerate elliptic equation

$$
\sum_{i, j=1}^{m} \frac{\partial}{\partial x_{j}}\left(p_{i j} \frac{\partial u}{\partial x_{i}}\right)-q u=f \quad \text { in } \Omega
$$

with the boundary condition

$$
u=\varphi \quad \text { on } \partial \Omega,
$$

where $\varphi \in W_{2}^{(1)}$.

We define a weak solution $u$ of this boundary value problem as follows :

If $u \in D W_{2}^{(1)}$ and if for any $\zeta \in D W_{2}^{(1)}$ we have

$$
D[u, \zeta]+H_{q}[u, \zeta]+(f, \zeta)=0,
$$

then we call $u$ weak solution of the problem (3)-(4).

Theorem 3.1. (Uniqueness). Assume that $\left(p_{i j}\right)=\left(p_{j i}\right)$ has the degeneracy of the $r$-th degree, and that 


$$
\begin{aligned}
& q \geqq 0, q \in L_{r_{1}} \quad\left(r_{1}=\left\{\begin{array}{cl}
\frac{m}{2-m r}(m \geqq 3 \text { or } m=2, r \neq 0) \\
\infty \quad(m=2, r=0) \\
1 \quad(m=1)
\end{array}\right)\right. \\
& f \in L_{r_{4}} \quad\left(r_{4}=\left\{\begin{array}{cl}
\frac{2 m}{m(1-r)+2} & (m \geqq 3 \text { or } m=2, r \neq 0) \\
2 & (m=2, r=0) \\
1 & (m=1)
\end{array}\right)\right. \\
& \varphi \in W_{2}^{(1)} \cap L_{r_{2}}\left(r_{2}=\left\{\begin{array}{cl}
\frac{2 m}{m(1+r)+2} & (m \geqq 3 \text { or } m=2, r \neq 0) \\
2 & (m=2, r=0) \\
\infty & (m=1)
\end{array}\right)\right.
\end{aligned}
$$

where $r$ is some constant with $0 \leqq r<\min \left\{\frac{2}{m}, 1\right\}$.

Then the weak solution of (3)-(4) is unique.

Proof. Let $u_{1}$ and $u_{2}$ be two weak solutions of (3)-(4). Then we have for any $\zeta \in D \dot{W}_{2}^{(1)}$

$$
\begin{aligned}
& D\left[u_{1}, \zeta\right]+H_{q}\left[u_{1}, \zeta\right]+(f, \zeta)=0 \\
& D\left[u_{2}, \zeta\right]+H_{q}\left[u_{2}, \zeta\right]+(f, \zeta)=0 .
\end{aligned}
$$

Therefore we have

$$
D\left[u_{1}-u_{2}, \zeta\right]+H_{q}\left[u_{1}-u_{2}, \zeta\right]=0 .
$$

Putting $\zeta=u_{1}-u_{2} \in D W_{2}^{(2)}$, because of $q \geqq 0$, we get

$$
D\left[u_{1}-u_{2}\right]=0 \text {. }
$$

From Lemmas 1.2 and 1.5 follows

$$
\begin{aligned}
& \left.\left\|u_{1}-\left.u_{2}\right|_{\left.\right|_{2} /(1+r)} ^{2} \leqq B_{m r}{ }^{2}\right\| \nabla\left(u_{1}-u_{2}\right)\right|_{i_{2} /(1+r)} ^{2} \\
& \leqq B_{m r^{\prime}}{ }^{2}\left|p_{0}^{-1}\right|_{i_{1} / r} D\left[u_{1}-u_{2}\right]=0 .
\end{aligned}
$$

Thus $u_{1}=u_{2}$ almost every where in $\Omega$.

Theorem 3.2. (Existence) Under the same assumptions as in Theorem 3.1, the problem (3)-(4) has a unique weak solution $u_{0}$ and moreover $u_{0}$ satisfies

$$
D\left[u_{0}\right]+H_{q}\left[u_{0}\right]+2\left(f, u_{0}\right)=\inf _{\substack{\varphi \\ D W_{2}^{(1)}}}\left(D[u]+H_{q}[u]+2(f, u)\right) .
$$


Proof. By Lemma 2.1

$$
\inf _{\substack{\varphi \\ D W_{2}^{(1)}}}\left(D[u]+H_{q}[u]+2(f, u)\right) \equiv d
$$

is finite. Taking a sequence $\left\{u_{k} \in C \stackrel{\varphi}{W}_{2}^{(1)}\right\}$ such that

$$
\lim _{k \rightarrow \infty}\left(D\left[u_{k}\right]+H_{q}\left[u_{k}\right]+2\left(f, u_{k}\right)\right)=d,
$$

we have

$$
\begin{aligned}
0 \leqq & \lim _{k, k^{\prime} \rightarrow \infty}\left(D\left[\frac{u_{k}-u_{k^{\prime}}}{2}\right]+H_{q}\left[\frac{u_{k}-u_{k^{\prime}}}{2}\right]\right) \\
= & \lim _{k, k^{\prime} \rightarrow \infty}\left\{\frac{1}{2}\left(D\left[u_{k}\right]+H_{q}\left[u_{k}\right]\right)+\frac{1}{2}\left(D\left[u_{k^{\prime}}\right]+H_{q}\left[u_{k^{\prime}}\right]\right)\right. \\
& \left.\quad-\left(D\left[\frac{u_{k}+u_{k^{\prime}}}{2}\right]+H_{q}\left[\frac{u_{k}+u_{k^{\prime}}}{2}\right]\right)\right\} \\
= & \lim _{k, k^{\prime} \rightarrow \infty}\left\{\frac{1}{2}\left(D\left[u_{k}\right]+H_{q}\left[u_{k}\right]+2\left(f, u_{k}\right)\right)\right. \\
& +\frac{1}{2}\left(D\left[u_{k^{\prime}}\right]+H_{q}\left[u_{k^{\prime}}\right]+2\left(f, u_{k^{\prime}}\right)\right) \\
& \left.\quad-\left(D\left[\frac{u_{k}+u_{k^{\prime}}}{2}\right]+H_{q}\left[\frac{u_{k}+u_{k^{\prime}}}{2}\right]+2\left(f, \frac{u_{k}+u_{k^{\prime}}}{2}\right)\right)\right\} \\
\leqq & \frac{1}{2} d+\frac{1}{2} d-d=0,
\end{aligned}
$$

i.e.,

$$
\lim _{k, k^{\prime} \rightarrow \infty} D\left[u_{k}-u_{k^{\prime}}\right]=H_{q}\left[u_{k}-u_{k^{\prime}}\right]=0 .
$$

Therefore there exists $u_{0} \in D \stackrel{\varphi}{W}(1)$ such that

$$
\lim _{k \rightarrow \infty} D\left[u_{0}-u_{k}\right]=\lim _{k \rightarrow \infty} H_{q}\left[u_{0}-u_{k}\right]=0,
$$

and thereafter from Lemma 1.7 follows

$$
\lim _{k \rightarrow \infty}\left(f, u_{0}-u_{k}\right)=0 .
$$

Thus we have

$$
\begin{aligned}
& D\left[u_{0}\right]+H_{q}\left[u_{0}\right]+2\left(f, u_{0}\right) \\
& =\lim _{k \rightarrow \infty}\left(D\left[u_{k}\right]+H_{q}\left[u_{k}\right]+2\left(f, u_{k}\right)\right)=d .
\end{aligned}
$$

Moreover for any $\zeta \in D W_{2}^{(1)}$ and any real number $\mu$ we have 


$$
\begin{aligned}
& \left(D\left[u_{0}+\mu \zeta\right]+H_{q}\left[u_{0}+\mu \zeta\right]+2\left(f, u_{0}+\mu \zeta\right)\right) \\
& \quad-\left(D\left[u_{0}\right]+H_{q}\left[u_{0}\right]+2\left(f, u_{0}\right)\right) \\
& =\mu^{2}\left(D[\zeta]+H_{q}[\zeta]\right)+2 \mu\left(D\left[u_{0}, \zeta\right]+H_{q}\left[u_{0}, \zeta\right]+(f, \zeta)\right) \geqq 0,
\end{aligned}
$$

and thereafter

$$
D[u, \zeta]+H_{q}[u, \zeta]+(f, \zeta)=0 .
$$

Hence $u_{0}$ is a weak solution of (3)-(4), which is a unique solution by Theorem 3.1.

Example 1. Consider the problem

$$
\begin{gathered}
\sum_{i=1}^{m} \frac{\partial}{\partial x_{1}}\left\{\left(2-\sqrt{\sum_{k=1}^{m} x_{k}^{2}}\right)^{r}\left(\sqrt{\sum_{k=1}^{m} x_{k}^{2}}-1\right)^{r} \frac{\partial u}{\partial x_{i}}\right\}-q u=f \quad \text { in } \Omega, \\
u=\varphi \quad \text { on } \partial \Omega,
\end{gathered}
$$

where

$$
\begin{aligned}
& \Omega=\left\{x \mid 1 \leqq \sum_{k=1}^{m} x_{k}{ }^{2} \leqq 2\right\}, \\
& q \geqq 0, q \in L_{r_{1}} \quad\left(r_{1}=\left\{\begin{array}{cl}
\frac{m}{2-m r}(m \geqq 3 \text { or } m=2, r \neq 0) \\
\infty \quad(m=2, r=0) \\
1 \quad(m=1)
\end{array}\right),\right. \\
& f \in L_{r_{4}} \quad\left(r_{4}=\left\{\begin{array}{cl}
\frac{2 m}{m(1-r)+2} & (m \geqq 3 \text { or } m=2, r \neq 0) \\
2 & (m=2, r=0) \\
1 & (m=1) \\
2 m & (m \geqq 3 \text { or } m=2, r \neq 0)
\end{array}\right),\right. \\
& \varphi \in W_{2}^{(1)} \cap L_{r_{2}}\left(r_{2}=\left\{\begin{array}{cl}
\frac{2 m=2, r=0)}{m(1+r)+2} & (m=1) \\
2 & \infty
\end{array}\right.\right.
\end{aligned}
$$

and $r$ is some constant with $0 \leqq r<\min \left\{\frac{2}{m}, 1\right\}$.

This problem has a unique weak solution.

Example 2. Consider the problem

$$
\begin{gathered}
\sum_{i=1}^{m} \frac{\partial}{\partial x_{i}}\left\{\left(3-\sqrt{\sum_{k=1}^{m} x_{k}^{2}}\right)^{r}\left|2-\sqrt{\sum_{k=1}^{m} x_{k}^{2}}\right|^{r}\left(\sqrt{\sum_{k=1}^{m} x_{k}^{2}}-1\right)^{r} \frac{\partial u}{\partial x_{i}}\right\} \\
-q u=f \quad \text { in } \Omega, \\
u=\varphi \quad \text { on } \partial \Omega,
\end{gathered}
$$


where

$$
\begin{aligned}
& \Omega=\left\{x \mid 1 \leqq \sqrt{\sum_{k=1}^{m} x_{k}^{2} \leqq}\right\}, \\
& q \geqq 0, q \in L_{r_{1}} \quad\left(r_{1}=\left\{\begin{array}{cl}
\frac{m}{2-m r}(m \geqq 3 \text { or } m=2, r \neq 0) \\
\infty \quad(m=2, r=0) \\
1 \quad(m=1)
\end{array}\right)\right. \\
& f \in L_{r_{4}} \quad\left(r_{4}=\left\{\begin{array}{cl}
\frac{2 m}{m(1-r)+2} & (m \geqq 3 \text { or } m=2, r \neq 0) \\
2 & (m=2, r=0) \\
\infty & (m=1)
\end{array}\right)\right. \\
& \varphi \in W_{2}^{(1)} \cap L_{r_{2}}\left(r_{2}=\left\{\begin{array}{cl}
2 m & (m \geq 3 \text { or } m=2, r \neq 0) \\
\frac{2 m}{m(1+r)+2} & (m=1)
\end{array}\right)\right.
\end{aligned}
$$

and $r$ is some constant with $0 \leqq r<\min \left\{\frac{2}{m}, 1\right\}$.

Again this problem has a unique weak solution.

\section{§4. Eigenvalue Problem}

In this section we treat the eigenvalue problem for the degenerate elliptic equation

$$
\sum_{i, j=1}^{m} \frac{\partial}{\partial x_{j}}\left(p_{i j} \frac{\partial u}{\partial x_{i}}\right)-q u+\lambda \rho u=0 \quad \text { in } \Omega
$$

with the boundary condition

$$
u=0 \quad \text { on } \partial \Omega \text {. }
$$

When $u$ is a non-trivial weak solution of (5)-(6), we call $u$ and $\lambda$ a weak eigenfunction and a weak eigenvalue for (5)-(6), respectively.

Theorem 4.1. (Discreteness) Assume that $\left(p_{i j}\right)=\left(p_{j i}\right)$ has the degeneracy of the $r$-th degree and that

$$
\begin{aligned}
& \rho, q \in L_{r_{3}}\left(r_{3}=\left\{\begin{array}{l}
\frac{m(2-r)}{2-m r}(m \geqq 2) \\
1+\varepsilon(\varepsilon \text { is some positive constant })(m=1)
\end{array}\right),\right. \\
& \rho \geqq \rho_{0}>0,
\end{aligned}
$$


where $r$ is some constant with $0 \leqq r<\min \left\{\frac{2}{m}, 1\right\}$.

Then the spectrum of the problem (5)-(6), i.e., the totality of the eigenvalues for (5)-(6) with respective multiplicity, is discrete.

Proof. Suppose on the contrary that we had a sequence of eigenvalues $\left\{\lambda_{n}\right\}$ such that $\lim _{n \rightarrow \infty} \lambda_{n}=\lambda_{0} \neq \infty$. Let $u_{n}$ be an eigenfunction corresponding to $\lambda_{n}$. For any $\zeta \in D W_{2}^{(1)}$ we have

$$
D\left[u_{n}, \zeta\right]+H_{q}\left[u_{n}, \zeta\right]-\lambda_{n} H\left[u_{n}, \zeta\right]=0 .
$$

If $\lambda_{n} \neq \lambda_{n^{\prime}}$ we get

$$
\begin{aligned}
& \lambda_{n} H_{\rho}\left[u_{n}, u_{n^{\prime}}\right]=D\left[u_{n}, u_{n^{\prime}}\right]+H_{q}\left[u_{n}, u_{n^{\prime}}\right] \\
& =D\left[u_{n^{\prime}}, u_{n}\right]+H_{q}\left[u_{n^{\prime}}, u_{n}\right]=\lambda_{n^{\prime}} H_{\rho}\left[u_{n^{\prime}}, u_{n}\right] \\
& =\lambda_{n^{\prime}} H_{\rho}\left[u_{n}, u_{n^{\prime}}\right] .
\end{aligned}
$$

Therefore we have

$$
H_{\rho}\left[u_{n}, u_{n^{\prime}}\right]=0 \text {. }
$$

Thus we can construct a system of eigenfunctions corresponding to $\left\{\lambda_{n}\right\}$ (we denote it again by $\left\{u_{n}\right\}$ ) such that

$$
H_{\rho}\left[u_{n}, u_{n^{\prime}}\right]= \begin{cases}1 & \left(n=n^{\prime}\right) \\ 0 & \left(n \neq n^{\prime}\right) .\end{cases}
$$

Then we have

$$
D\left[u_{n}\right]+H_{q}\left[u_{n}\right]=\lambda_{n} H_{\rho}\left[u_{n}\right]=\lambda_{n} .
$$

From the boundedness of $\left\{\lambda_{n}\right\}$ results the boundedness of $D\left[u_{n}\right]+H_{q}\left[u_{n}\right], D\left[u_{n}\right]$ (cf. Lemma 2. 3), $\left\|\nabla u_{n}\right\|_{2 /(1+r)}$ (cf. Lemma 1.5), and thereafter $\left\|u_{n}\right\|_{2 /(1+r)}$ (cf. Lemma 1.2). Hence from Lemma 1.4 it follows that $\left\{u_{n}\right\}$ is relatively compact in $L_{2}$. Taking a Cauchy sequence in $L_{2}$ from $\left\{u_{n}\right\}$ (we denote it again by $\left\{u_{n}\right\}$ ), we have

$$
\left.H_{\rho}\left[u_{n}-u_{n^{\prime}}\right] \rightarrow 0 \quad\left(n, n^{\prime} \rightarrow \infty\right) \text { (cf. Lemma 1. } 6^{\prime}\right) .
$$

On the other hand by the selection of $\left\{u_{n}\right\}$ we have

$$
H_{\rho}\left[u_{n}-u_{n^{\prime}}\right]=H_{\rho}\left[u_{n}\right]-2 H_{\rho}\left[u_{n}, u_{n^{\prime}}\right]+H_{\rho}\left[u_{n^{\prime}}\right]=2>0 .
$$

This is a contradiction. 
Theorem 4.2. (Existence). Under the same assumptions as in Theorem 4.1, the eigenvalue problem for (5)-(6) has weak eigenfunctions $u_{1}, u_{2}, \cdots, u_{n}, \cdots$ corresponding to weak eigenvalues $\lambda_{1} \leqq \lambda_{2} \leqq \cdots$ $\leqq \lambda_{m} \leqq \cdots$, and moreover $u_{n}$ satisfies

where

$$
D\left[u_{n}\right]+H_{q}\left[u_{n}\right]=\inf _{\mathfrak{w}_{n}}\left(D[u]+H_{q}[u]\right)=\lambda_{n},
$$

$$
\mathfrak{B}_{n}=\left\{u \in D \dot{W}_{2}^{(1)} \mid H_{\rho}[u]=1, H_{\rho}\left[u, u_{n^{\prime}}\right]=0\left(n^{\prime}=1, \cdots, n-1\right)\right\} .
$$

Proof. By Lemma 2.2

$$
\inf _{\mathfrak{w}_{n}}\left(D[u]+H_{q}[u]\right) \equiv \lambda_{n}
$$

is finite. Taking some sequence $\left\{u_{n k} \in \mathfrak{w}_{n}\right\}$ such that

$$
\lim _{k \rightarrow \infty}\left(D\left[u_{n k}\right]+H_{q}\left[u_{n k}\right]\right)=\lambda_{n},
$$

we gain the boundedness of $D\left[u_{n k}\right]+H_{q}\left[u_{n k}\right], D\left[u_{n k}\right]$ (cf. Lemma 2.3), $\left\|\nabla u_{n k}\right\|_{2 /(1+r)}$ (cf. Lemma 1.5) and thereafter $\left\|u_{n k}\right\|_{2 /(1+r)}$ (cf. Lemma 1.2). From Lemma 1.4 follows the relative compactness of $\left\{u_{n k}\right\}$. Taking a Cauchy sequence in $L_{2}$ from $\left\{u_{n k}\right\}$ (we denote it again by $\left.\left\{u_{n k}\right\}\right)$, we have

$$
\lim _{k, k^{\prime} \rightarrow \infty}\left\|u_{n k}-u_{n k^{\prime}}\right\|_{2}=0
$$

and thereafter

$$
\lim _{k, k^{\prime} \rightarrow \infty} H_{\rho}\left[u_{n k}-u_{n k^{\prime}}\right]=\lim _{k, k^{\prime} \rightarrow \infty} H_{q}\left[u_{n k}-u_{n k^{\prime}}\right]=0
$$

(cf. Lemma 1.6'). Therefore for any positive number $\varepsilon$ and for large $k \geqq k_{0}(\varepsilon)$ we have

$$
\begin{aligned}
& H_{\rho}\left[\frac{u_{n k}-u_{n k^{\prime}}}{2}\right]<\frac{\varepsilon}{4}, \\
& 1 \geqq H_{\rho}\left[\frac{u_{n k}-u_{n k^{\prime}}}{2}\right]=\frac{1}{2} H_{\rho}\left[u_{n k}\right]+\frac{1}{2} H_{\rho}\left[u_{n k^{\prime}}\right]-H_{\rho}\left[\frac{u_{n k}-u_{n k^{\prime}}}{2}\right] \\
& >1-\frac{\varepsilon}{4}
\end{aligned}
$$

and

$$
\begin{aligned}
& D\left[\frac{u_{n k}+u_{n k^{\prime}}}{2}\right]+H_{q}\left[\frac{u_{n k}+u_{n k^{\prime}}}{2}\right] \geqq \lambda_{n} H_{\mathrm{\rho}}\left[\frac{u_{n k}+u_{n k^{\prime}}}{2}\right] \\
& \geqq \min \left\{\lambda_{n}, \lambda_{n}\left(1-\frac{\varepsilon}{4}\right)\right\} .
\end{aligned}
$$


Thus we have

$$
\begin{aligned}
& 0 \leqq D\left[\frac{u_{n k}-u_{n k^{\prime}}}{2}\right]+H_{q}\left[\frac{u_{n k}-u_{n k^{\prime}}}{2}\right]=\frac{1}{2}\left(D\left[u_{n k}\right]+H_{q}\left[u_{n k}\right]\right) \\
& +\frac{1}{2}\left(D\left[u_{n k^{\prime}}\right]+H_{q}\left[u_{n k^{\prime}}\right]\right)-\left(D\left[\frac{u_{n k}+u_{n k^{\prime}}}{2}\right]+H_{q}\left[\frac{u_{n k}+u_{n k^{\prime}}}{2}\right]\right) \\
& <\frac{\lambda_{n}+\varepsilon}{2}+\frac{\lambda_{n}+\varepsilon}{2}-\min \left\{\lambda_{n}, \lambda_{n}\left(1-\frac{\varepsilon}{4}\right)\right\}=\max \left\{\varepsilon, \varepsilon\left(1+\frac{\lambda_{n}}{4}\right)\right\} .
\end{aligned}
$$

or

$$
0 \geqq D\left[\frac{u_{n k}-u_{n k^{\prime}}}{2}\right]+H_{q}\left[\frac{u_{n k}-u_{n k^{\prime}}}{2}\right] \geqq \lambda_{n} H\left[\frac{u_{n k}-u_{n k^{\prime}}}{2}\right] \geqq \frac{\varepsilon \lambda_{n}}{4},
$$

i.e., we have

$$
\frac{\varepsilon \lambda_{n}}{4} \leqq D\left[\frac{u_{n k}-u_{n k^{\prime}}}{2}\right]+H_{q}\left[\frac{u_{n k}-u_{n k^{\prime}}}{2}\right] \leqq \varepsilon \max \left\{1,1+\frac{\lambda_{n}}{4}\right\} .
$$

Thus we have

$$
\begin{aligned}
& \lim _{k, k^{\prime} \rightarrow \infty} D\left[u_{n k}-u_{n k^{\prime}}\right]=\lim _{k, k^{\prime} \rightarrow \infty} H_{q}\left[u_{n k}-u_{n k^{\prime}}\right] \\
& =\lim _{k, k^{\prime} \rightarrow \infty} H_{\rho}\left[u_{n k}-u_{n k^{\prime}}\right]=0 .
\end{aligned}
$$

This implies that there exists $u_{n} \in \mathfrak{W}_{n}$ and that $u_{n}$ satisfies

$$
D\left[u_{n}\right]+H_{q}\left[u_{n}\right]=\lambda_{n} .
$$

Moreover for any $\zeta \in \mathfrak{W}_{n}$ and any real number $\mu$ we have

$$
\begin{aligned}
D & {\left[u_{n}+\mu \zeta\right]+H_{q}\left[u_{n}+\mu \zeta\right]-\lambda_{n} H_{\rho}\left[u_{n}+\mu \zeta\right] } \\
= & \mu^{2}\left(D[\zeta]+H_{q}[\zeta]-\lambda_{n} H_{\rho}[\zeta]\right)+2 \mu\left(D\left[u_{n}, \zeta\right]\right. \\
& \left.+H_{q}\left[u_{n}, \zeta\right]-\lambda_{n} H_{\rho}\left[u_{n}, \zeta\right]\right)+\left(D\left[u_{n}\right]+H_{q}\left[u_{n}\right]-\lambda_{n} H_{\rho}\left[u_{n}\right]\right) \\
= & \mu^{2}\left(D[\zeta]+H_{q}[\zeta]-\lambda_{n} H_{\rho}[\zeta]\right)+2 \mu\left(D\left[u_{n}, \zeta\right]\right. \\
& \left.+H_{q}\left[u_{n}, \zeta\right]-\lambda_{n} H_{\rho}\left[u_{n}, \zeta\right]\right) \geqq 0
\end{aligned}
$$

and therefore we have

$$
D\left[u_{n}, \zeta\right]+H_{q}\left[u_{n}, \zeta\right]-\lambda_{n} H_{\rho}\left[u_{n}, \zeta\right]=0 .
$$

Now for any $\zeta \in D W_{2}^{(1)}$ set

$$
\eta=\zeta-\sum_{n^{\prime}=1}^{n-1} u_{n^{\prime}} H_{\rho}\left[u_{n^{\prime}}, \zeta\right] .
$$

Since

$$
\left(H_{\rho}[\eta]\right)^{-1 / 2} \eta \in \mathfrak{W}_{n},
$$


we have

$$
\begin{aligned}
& D\left[u_{n}, \zeta\right]+H_{q}\left[u_{n}, \zeta\right]-\lambda_{n} H_{\rho}\left[u_{n}, \zeta\right] \\
& =D\left[u_{n}, \eta\right]+H_{q}\left[u_{h}, \eta\right]-\lambda_{n} H_{\rho}\left[u_{n}, \zeta\right] \\
& \quad+\sum_{n^{\prime}=1}^{n-1} H_{\rho}\left[u_{n}, \zeta\right]\left(D\left[u_{n}, u_{n^{\prime}}\right]+H_{q}\left[u_{n}, u_{n^{\prime}}\right]-\lambda_{n} H_{\rho}\left[u_{n}, u_{n^{\prime}}\right]\right) \\
& =D\left[u_{n}, \eta\right]+H_{q}\left[u_{n}, \eta\right]-\lambda_{n} H_{\rho}\left[u_{n}, \zeta\right] \\
& \quad+\sum_{n^{\prime}=1}^{n-1} H_{\rho}\left[u_{n^{\prime}}, \zeta\right]\left(D\left[u_{n^{\prime}}, u_{n}\right]+H_{q}\left[u_{n^{\prime}}, u_{n}\right]-\lambda_{n^{\prime}} H_{\rho}\left[u_{n^{\prime}}, u_{n}\right]\right) \\
& =0 .
\end{aligned}
$$

Example 1. Consider the eigenvalue problem for

$$
\begin{gathered}
\sum_{i=1}^{m} \frac{\partial}{\partial x_{i}}\left\{\left(2-\sqrt{\sum_{k=1}^{m} x_{k}^{2}}\right)^{r}\left(\sqrt{\sum_{k=1}^{m} x_{k}^{2}}-1\right)^{r} \frac{\partial u}{\partial x_{i}}\right\} \\
-q u+\lambda \rho u=0 \quad \text { in } \Omega, \\
u=0 \quad \text { on } \partial \Omega,
\end{gathered}
$$

where

$$
\begin{aligned}
& \Omega=\left\{x \mid 1<\sqrt{\sum_{k=1}^{m} x_{k}^{2}}<2\right\}, \\
& \rho, q \in L_{r_{3}}\left(r_{3}=\left\{\begin{array}{c}
\frac{m(2-r)}{2-m r}(m \geqq 2) \\
1+\varepsilon(\varepsilon \text { is some positive constant })(m=1)
\end{array}\right),\right. \\
& \rho \geqq \rho_{0}>0
\end{aligned}
$$

and $r$ is some constant with $0 \leqq r<\min \left\{\frac{2}{m}, 1\right\}$.

This problem has weak eigenvalue and its spectrum is discrete.

Example 2. Consider the eigenvalue problem for

$$
\begin{gathered}
\sum_{i=1}^{m} \frac{\partial}{\partial x_{i}}\left\{\left(3-\sqrt{\sum_{k=1}^{m} x_{k}^{2}}\right)^{r}\left|2-\sqrt{\sum_{k=1}^{m} x_{k}^{2}}\right|^{r}\left(\sqrt{\sum_{k=1}^{m} x_{k}^{2}}-1\right)^{r} \frac{\partial u}{\partial x_{i}}\right\} \\
-q u+\lambda \rho u=0 \quad \text { in } \Omega, \\
u=0 \quad \text { on } \partial \Omega,
\end{gathered}
$$

where

$$
\Omega=\left\{x \mid 1<\sqrt{\sum_{k=1}^{m} x_{k}^{2}}<3\right\}
$$




$$
\begin{aligned}
& \rho, q \in L_{r_{3}}\left(r_{3}=\left\{\begin{array}{l}
\frac{m(2-r)}{2-m r}(m \geqq 2) \\
1+\varepsilon(\varepsilon \text { is some positive canstant })(m=1)
\end{array}\right),\right. \\
& \rho \geqq \rho_{0}>0
\end{aligned}
$$

and $r$ is some constant with $0 \leqq r<\min \left\{\frac{2}{m}, 1\right\}$.

This problem also has weak eigenvalues and its spectrum is discrete.

\section{References}

[1] Nirenberg, L., On elliptic partial equations, Ann. Scuola Norm. Sup. Pisa, ser. 3, 13 (1959), 115-162.

[2] Sobolev, S. L., Some applications of functional analysis to mathematical physics, Izdatel'stvo Sibirskogo Otdaleniya AN SSSR, Novosibirsk, 1962. (Russian)

[3] Suzuki, K., The first boundary value problem and the first eigenvalue problem for the elliptic equations degenerate on the boundary, Publ. RIMS Kyoto Univ. Ser. A, 3 (1967-8), 299-335. 